\title{
IV. Litauer und Juden
}

Der jüdisch-litauische Gegensatz stellte nicht die Eskalation bereits lang angelegter Konfliktlinien dar. Während der Zwischenkriegszeit herrschte nämlich ein relativ entspanntes Verhältnis zwischen der litauischen Staatsnation und der jüdischen Minderheit. Dies hatte nicht zuletzt mit den polnisch-litauischen Spannungen um Vilnius zu tun, die aus litauischer Sicht die Juden zu einer nicht unbedeutenden ethnischen Gruppe gemacht hatten, von der man sich Unterstützung beim Kampf um die historische Hauptstadt erhoffte. Für die jüdische Bevölkerung hatte dies in den ersten Jahren der litauischen Republik die Folge, dass in dem neuentstandenen baltischen Staat vorbildliche Minderheitenrechte existierten, die den Juden Freiheiten ermöglichten wie nie zuvor in der Geschichte dieser Region. Sogar ein eigenes Ministerium für jüdische Angelegenheiten war gegründet worden. ${ }^{1}$

Vilnius ist als Sonderfall zu betrachten, denn die dortigen ethnischen Verhältnisse unterschieden sich von denen in Kaunas und Šiauliai aufgrund der historischen Entwicklung der Stadt. Sie war seit langem vor allem durch ihre polnischund jiddischsprachige Bevölkerung geprägt worden, während die Litauer trotz ihrer historischen Ansprüche auf die Stadt an der Neris deutlich in der Minderheit waren. Daran hatte sich prinzipiell auch nach der Übernahme der Stadt durch Litauen im Oktober 1939 wenig geändert. ${ }^{2}$ Bei der Volkszählung im Mai 1942 hatten sich bei einer ,arischen' Bevölkerung von 144000 Personen rund 31000 Befragte $(21,71 \%)$ als litauischsprachig bezeichnet. Demgegenüber gaben mehr als 100000 Bewohner $(70,91 \%)$ bei der Frage nach der Volkszugehörigkeit polnisch an. ${ }^{3}$

Den deutschen Besatzern waren die besonderen ethnischen Verhältnisse in Vilnius wohl bewusst („ein heikles und schwieriges Gebiet“4), weswegen man ver-

1 Grundlegend, Liekis, State, passim; Vareikis, Antisemitizmas, passim. Zur Vorgeschichte Tauber, Alliies, passim. Vgl. dazu die Aussage des Rabbiners Dr. Bloch, der als Lehrer an jüdischen Gymnasien und Schulen tätig war, vom 17. Juli 1959, in: EK 3 Verfahren, Bd. 3, Bl. 889. Ähnlich die Aussage von Vytautas Bernotas, der in Mazeikiai aufwuchs. Vgl. EK 3 Verfahren, Bd. 5, Bl. 1875, Aussage von Vytautas Bernotas. Zur Position des Präsidenten Smetona Truska, Smetona, S. $296 \mathrm{ff}$.

2 Viele Juden aus dem deutsch besetzten Teil Polens flohen nach Vilnius, darunter auch Herman Kruk, Last Days, S. 1 ff.

3 LCVA R-643, ap. 3, b. 197, Bl. 15, Vorder- und Rückseite, Leiter des statistischen Büros der Stadt Wilna an den Herrn Kommandanten der Feldkommandantur des Bereiches Wilna, Betr.: Zählung vom 27. Mai 1942 vom 9. Juli 1943. Wie problematisch diese Eigenzuschreibungen waren, ergibt sich aus dem Schreiben: „Es ist zu bemerken, dass 21363 Personen $(=18,88 \%)$ sich zwar nicht als Litauer, jedoch als litauischer Sprache kundig [sic!] bezeichnet haben. Nun wurde im Oktober 1941 bei der Verteilung der Lebensmittelkarten auch eine Zählung der Einwohner durchgeführt. Damals hatten sich 52370 Personen als Litauer gemeldet. Es ist sehr bemerkenswert, dass man praktisch genau dieselbe Zahl bekommt, wenn man die Zahl der Litauer nach der Volkszählung vom 27. Mai 1942 mit der erwähnten Zahl der die litauische Sprache beherrschenden Nichtlitauer addiert." Weitere Zahlen zur ethnischen Zusammensetzung des Gebietes bei Dieckmann, Besatzungspolitik, 1, S. $281 \mathrm{f}$.

4 LCVA R-677, ap. 2, b. 92, Bl. 8, Feldkommandantur 814, gez. Zehnpfenning, Betr.: Befriedung des Gebietes, Bericht vom 29. Juli 1941. 
suchte, die litauischen Ansprüche und Lituanisierungsmaßnahmen ${ }^{5}$ nicht überhandnehmen zu lassen. Bereits in einer Bekanntmachung vom 16. Juli 1941 hieß es z. B.: „Litauer, Weißrussen und Polen haben das gleichmäßige Recht in- und außerhalb der beruflichen Arbeit ihre Muttersprache zu sprechen; es besteht kein Anlaß, dass der eine Bevölkerungsteil die Sprache des anderen erlernt." ${ }^{\text {"6 Inwie- }}$ weit sich das Verhalten der polnischen Bevölkerung von dem der litauischen unterschied, lässt sich aufgrund der Quellenlage nur schwer feststellen. Oft ist von Einheimischen oder Christen die Rede, vereinzelt finden sich Hinweise darauf, dass die Polen mehr Sympathie mit den Juden erkennen ließen als die Litauer. ${ }^{7}$ Im Folgenden werde ich mich daher vor allem auf Beispiele aus dem litauischen Kernland oder eindeutiger ethnischer Zuordnung beziehen, um die jüdisch-litauischen Beziehungen zu verdeutlichen.

Ein nicht zu unterschätzendes Motiv für das Verhalten von Litauern in den ersten Kriegstagen war die Aussicht auf Beute. So berichtet Gustav Hörmann, bei einer Besprechung über das Schicksal der im Herbst 1941 noch lebenden Juden, an der alle führenden Persönlichkeiten in Kaunas teilnahmen, habe sich der litauische Vertreter Kubiliūnas "gegen die Erhaltung der Juden“ ausgesprochen, „da die Litauer es [sc. die „Nicht-Erhaltung“ - JT] wahrscheinlich wünschten und angeblich die Wohnräume in Wyliampole [sic!] benötigten." ${ }^{\text {" Auch der Rabbiner }}$ Dr. Bloch sah dieses Motiv als wichtig an: „Die Litauer haben zweifellos deshalb sich an den Vernichtungsmaßnahmen beteiligt, weil sie sich an dem jüdischen Eigentum bereichern durften. ${ }^{\text {"9 }}$ Chana Ralbè verlor bei der Umsiedlung ins Ghetto fast ihre gesamte Habe, weil sich der für den Umzug angeworbene litauische Bauer mit dem vollgeladenen Viehwagen aus dem Staub machte. ${ }^{10}$ Jüdische Wohnungen und Mobilar in Vilnius gingen Ende August 1941 an Männer der litauischen Sicherheitspolizei über ${ }^{11}$, die sich darum bewarben. ${ }^{12}$ Und auch die

${ }^{5}$ Vgl. LCVA R-677, ap. 2, b. 92, Bl. 8, Feldkommandantur 814, gez. Zehnpfennning, Betr.: Befriedung des Gebietes, Bericht vom 29. Juli 1941.

${ }^{6}$ Den Besatzern schwebte vor allem die Erlernung des Deutschen vor. Vgl. LCVA R-677, ap. 2, b. 91, Bl. 13, Deutsche Kommandobehörde Wilna, Bekanntmachung vom 16. Juli 1941.

7 Vor allem Herman Kruk unterschied zwischen den ethnischen Gruppen. Z. B. Kruk, Last Days, S. 110, S. 352. Im April 1943 stellte Kruk, Last Days, S. 523f., S. 531, ein Anwachsen des Antisemitismus unter der polnischen Bevölkerung fest, das er auch auf die Entdeckung des Massengrabes der von den Sowjets ermordeten polnischen Offiziere bei Katyn zurückführte.

${ }^{8}$ EK 3 Verfahren, Bd. 3, Bl. 799, Aussage Gustav Hörmann vom 2. September 1946 (Abschrift).

9 Vgl. Aussage Bloch vom 17. Juli 1959, EK 3 Verfahren, Bd. 3, Bl. 889, Bl. 890.

10 Vgl. LCVA R-973, ap. 1, b. 7, Bl. 497, Chana Ralbe an die Kriminalpolizei in Kauen durch die Jüdische Ghettopolizei in Marijampolè vom 24. August 1941. Eine weitere Schilderung des Verhaltens von Litauern in den Tagen der Ghettoisierung bei Tory, Surviving, S. $24 \mathrm{ff}$.

${ }^{11}$ LCVA R-614, ap. 1, b. 285, Bl. 177f, Litauische Sicherheitspolizei Wilna-Bezirk, Chef des Bezirkes A. Lileikis an den Chef der Sicherheitspolizei und des SD vom 27. August 1941. Die Quartiere wurden möbliert und mit Küche gewünscht. Murer genehmigte am 1. September 1941 diese Form des Wohnungserwerbs für sieben der zehn benannten litauischen SD-Polizisten.

12 Vgl. LCVA R-614, ap. 1, b. 204, Bl. 5, Polizist der Preisüberwachungsstelle Jonas Gumbaragis an den Herrn Polizeichef, eingegangen am 16. Oktober 1941. Ein weiteres Beispiel ebd., Bl. 6, Stadtpolizei Wilna, Stellvertreter des Revierkommissars des 2. Polizeireviers an den Herrn Revierführer vom 23. September 1941. 
Universität wollte so schnell wie möglich über bisher von Juden bewohnte Räume verfügen. ${ }^{13}$ Die persönliche Bereicherung konnte auch Rettung bedeuten: Abraham Sutzkever war in der Nacht auf den 28. Oktober 1941 aus dem Ghetto Vilnius geflohen und stieß auf Litauer: „Zwei Polizisten verstellen uns mit den Gewehren den Weg. Wir haben keine andere Wahl - ich erzähle ihnen die Wahrheit: wir sind aus dem Getto geflohen. Ich nehme ein Paar Stiefelschäfte heraus, die ich aus dem Getto mitgenommen habe, und drücke sie einem von ihnen in die Hand. Das Geschenk ist offenbar ganz nach seinem Herzen. Er rät uns auf der Nowigorod [Straße - JT] zu gehen: Dort werden wir keine Patrouillen antreffen." 14 Bereits bei der Ghettoisierung am 6. September 1941 gelangten Juden beim Geleit durch litauische Polizisten nicht ins Ghetto, sondern ins berüchtigte Gefängnis Lukiškès. Man konnte sich allerdings für 30 RM oder andere Wertgegenstände bei der litauischen Wache und den Partisanen den Weg ins Ghetto erkaufen. ${ }^{15}$

Einfache Bürger wandten sich an das städtische Wohnungsamt mit Bitten um Überlassung von jüdischen Möbeln. Mancher war mit seiner Neuerwerbung unzufrieden wie etwa Stasys Grušas, der aus dem jüdischen Lager der Behörde einen Schrank für 70 Rubel erworben hatte, dem die mittlere Tür fehlte. Da ein Ersatz weitaus teurer zu Buche schlug, als der Käufer erwartet hatte, bat er um einen Preisnachlass von 20 Rubel (der ihm auch gewährt wurde). ${ }^{16}$ Jonas Pigega, der unter den Sowjets im Gefängnis gesessen hatte und nach dem deutschen Überfall sofort den litauischen Partisanen beigetreten war, erhoffte sich aufgrund seiner politischen Tätigkeit einen Rabatt. ${ }^{17}$ Auch junge Ehepaare waren an jüdischem Mobiliar interessiert. ${ }^{18}$ Und Witold Bohdanowiecz, dem Namen nach polnischer Abstammung, wandte sich gleich direkt an den Gebietskommissar und bat um Hilfe gegen einen Hausverwalter, der ein ausgeprägtes Interesse an den Möbeln der jüdischen Wohnung zeigte, die dem ausgebombten Bohdanowiecz zugewiesen worden waren. ${ }^{19}$ Doch nicht nur Mobiliar, auch ganze Läden wechselten den

${ }^{13}$ Vgl. LCVA R-614, ap. 1, b. 204, Bl. 12, Vilniaus miesto sekretoriatas [Sekretariat der Stadt Vilnius] an den Herrn Gebietskommissar der Stadt Wilna, Betr.: Abfahren der jüdischen Sachen vom 22. Oktober 1941. Um die Bedeutung der Angelegenheit zu unterstreichen, trägt das Schreiben die Unterschrift des Bürgermeisters der Stadt Vilnius Dabulevičius. Ebd., Bl. 13, findet sich ein „Verzeichnis der Juden, die früher in den Universitätsräumen wohnten oder ein Geschäft hatten". Aufgeführt sind 15 Namen.

${ }^{14}$ Sutzkever, Geto, S. 66.

${ }^{15}$ Vgl. den Bericht bei Kaczerginski, Hurbn, S. 142 f.

${ }^{16}$ LCVA R-614, ap. 1, b. 374, Bl. 4, VMS Butu ir turtų eksploatacijos VI Apylinkès Vedijui [An den Leiter der Wohnungs- und Besitzabteilung des VI. Bezirks der Stadtverwaltung Vilnius] vom 7. Januar 1942. Ähnlich das Schreiben von Jurgis Matoré, der um nochmalige Überprüfung des festgesetzten Preises bat, da er ihm zu hoch erschien. LCVA R-614, ap. 1, b. 374, Bl. 25, P. Butų ir turtų skyriaus viršininko [An den Herrn Vorsitzenden der Wohnungs- und Besitzabteilung] (ohne Datum).

${ }^{17}$ LCVA R-614, ap. 1, b. 374, Bl. 26, Vil. M. S. Butų ir turtų skyriaus viršininko [An den Vorsitzenden der Wohnungs- und Besitzabteilung der Stadtverwaltung Vilnius] vom 15. Dezember 1941.

18 Vgl. LCVA R-614, ap. 1, b. 381, Bl. 447, Alexander Rudys an den Herrn Gebietskommissar der Stadt Wilna, Gesuch vom 17. Dezember 1941. Dem Gesuch wurde entsprochen.

${ }^{19}$ LCVA R-614, ap. 1, b. 374, Bl. 33, Herrn Gebietskommissar Stadt Wilna vom 17. Februar 1942. Das Schreiben hatte nicht die gewünschte Wirkung, denn am 3. März 1942 wandte sich Bohdanowiecz erneut an den Gebietskommissar (ebd., Bl. 34). 
Besitzer. ${ }^{20}$ In den Akten hat sich eine dementsprechende Auflistung mit der für sich sprechenden Überschrift „Verzeichnis der Antragsteller für die Einsetzung in die ehem. jüdischen Werkstätten und Betriebe " für Vilnius erhalten. ${ }^{21}$ Zumindest teilweise hatte die Verwaltung den Überblick über die Aneignung jüdischen Besitzes verloren ${ }^{22}$, weswegen Ende 1941 in Vilnius dreisprachige Meldeformulare kursierten, in die ehemals in jüdischen Händen befindliche Gegenstände eingetragen werden mussten. ${ }^{23}$ Die Sprachregelung lautete: „Verzeichnis der für zeitweilige Benutzung übergebenen Habe“. ${ }^{24}$ In Einzelfällen kam es sogar zu Beschwerden von litauischer Seite, weil sich Deutsche bedienten. ${ }^{25}$ Der Kreis derer, die auf diese Weise Nutznießer der Massenmorde wurden, war weit größer als der der eigentlichen Täter. ${ }^{26}$

Für die jüdische Erfahrung waren diese ersten Tage der deutschen Herrschaft fundamental. Levi Shalit spricht zutreffend davon, die Juden in Šiauliai seien Menschen außerhalb jeden Rechtes gewesen, die sich selbst nicht hätten beschützen können und auch niemanden gehabt hätten, an den man sich hätte wenden können. ${ }^{27}$ Das Blatt „Tevyne““ (Vaterland) forderte bereits am 10. Juli die „Reinigung“ der Stadt von Juden. ${ }^{28}$ In vielen Berichten bleiben daher die ersten Tage der Besetzung in besonders schrecklicher Erinnerung. ${ }^{29}$ Melech Gold, der sich als blauäugiger blonder Mann als ,Arier' auf die Straßen von Kaunas wagte, erinnerte sich noch im Jahre 1983 an die Vorgänge: „Ich habe etwa am dritten Tag nach dem deutschen Einmarsch Litauer in Aktion gesehen. Es waren unorgani-

20 LCVA R-614, ap. 1, b. 381, Bl. 249, Kostas Vasilauskas an das Gebietskommissariat in WilnaStadt vom 7. November 1942: „Im Dezember 1941 übernahm ich nach den Juden ein Geschäft bei der Wilnaerstraße 24-6. Im Geschäft [...] befand sich damals 2 Ladenbüffet und zwei Ladentische [...] Da ich nun schon ein Jahr in dem Geschäft arbeite, da ich das Ladeninventar schonte und mir auf meine eigenen Kosten es ausbesserte, möchte ich es gern nun zum Eigentum kaufen." Vgl. auch ebd., Bl. 240, das Gesuch des Schusters Andreius Žukovski, der 1941 zwei jüdische Ladentische erhalten hatte.

21 Vgl. LCVA R-643, ap. 3, b. 11, Bl. 133 ff. Der Großteil der Verzeichnisse ist in litauischer Sprache, insgesamt werden 285 Betriebe aufgeführt, vom Inventar eines Zahnarztes bis zu einer Hausnäherei. Sehr oft wurde die Verfügungsgewalt über die Läden auch der Stadtverwaltung übertragen. Die Namen der Anwärter sind ihrer sprachlichen Herkunft nach Litauisch und nicht Polnisch.

22 Auch Murer war nicht zufrieden und bemängelte die übereilte Auflösung jüdischer Werkstätten. Vgl. LCVA R-643, ap. 3, b. 11, Bl. 236, Der Gebietskommissar der Stadt Wilna i. A. Murer an die Stadtverwaltung Wilna, Eingangsstempel vom 14. Oktober 1941.

${ }^{23}$ Ein Beispiel in LCVA R-614, ap. 1, b. 381, Bl. 589.

24 Vgl. LCVA R-614, ap. 1, b. 381, Bl. 591.

25 LCVA R-1534, ap. 1, b. 191, Bl. 420, Amtsvorsteher des Bezirks Seredžiai an den Kreischef Kauen vom 18. September 1941: „Ich bitte Sie Schritte zu unternehmen, dass in dem Städtchen das Entwenden von jüdischem Besitz dem deutschen Militär verboten würde [sic!] .“ Offensichtlich änderte sich nichts. Vgl. ebd., Bl. 469, undatiert.

26 Darauf zielt Jeruschalmi, Umkum, S. 1830, mit der lakonischen Bemerkung, viele Litauer hätten sich zwar direkter Aktionen enthalten, aber die Resultate der Judenausrottung "genossen“.

27 Vgl. Shalit, Azoy, S. 30.

28 Vgl. Jeruschalmi, Umkum, S. 1771.

29 Vgl. z. B. EK 3 Verfahren, Bd. 1, Bl. 177b und 177c, Schreiben von Rosa Simon an die Redaktion der Jedioth Chadaschoth vom 10. Dezember 1958; vgl. auch Gringauz, Hurbn 7, S. 11. Zu den Morden zudem Gefen, Funke, S. 17. 
sierte Banden in litauischen Militäruniformen oder nur in Teilen von Uniformen, bewaffnet mit Gewehren, Pistolen, Handgranaten. Sie drangen in jüdische Behausungen, raubten, schlugen auf die Juden zu [sic!] und beschimpften wild die überfallenen Juden [...] Sie heulten judenfeindlichen Parollen [sic!] und schlugen die Juden. Sie schleppten von den jüdischen Wohnungen, was ihnen gefiel. “30 Deutsche Zuschauer machten in Vilnius ähnliche Beobachtungen ${ }^{31}$, während in Kaunas die Schrecken des VII. Forts in besonderer Weise mit litauischen Tätern verbunden waren. ${ }^{32}$ „Es mag am 24./25. Juni gewesen $\operatorname{sein}^{33}$, als durch einheimische Litauer, sogen. Polizei, die ersten Pogrome gegen die Juden durchgeführt wurden. Ich habe selbst gesehen, wie laufend Trupps in Stärke von 30-40 Mann unter litauischer Bewachung durch die Stadt und auf ein Fort getrieben wurden. Diese Juden wurden durch die Litauer auf dem 7. Fort liquidiert. Die Schüsse habe ich zum Teil selbst gehört. "34 Schließlich mussten sogar die litauischen Wachen abgezogen werden, weil es Tag und Nacht zu Morden kam und die in der Nähe liegenden Wehrmachteinheiten protestierten. ${ }^{35}$

Alltäglich war ein Vorfall wie jener vom 24. Juli 1941, bei dem jüdische Arbeiter „durch litauische Offizielle“ festgenommen wurden und nur durch eine Intervention deutscher Soldaten wieder befreit werden konnten. ${ }^{36}$ Am 6. Dezember 1941 schlug Juozas Sudorgas mit einer Peitsche Juden, die sich auf dem Weg von ihrem Arbeitsplatz ins Ghetto befanden. Zur Anzeige durch einen deutschen Soldaten gebracht, fiel die Strafe recht milde aus: 15 RM Geldstrafe bzw. drei Tage Arrest. ${ }^{37}$ Teilweise hoffte man in den ersten Kriegstagen sogar, die Deutschen würden die litauische Willkür beenden. In Šiauliai gab es bei einer Versammlung der Juden im Gymnasium Stimmen, die sich dafür aussprachen, alle Juden sollten sich in zwei Gebäuden vor den hasserfüllten Litauern verbarrikadieren, bis die Deutschen die Stadt einnehmen würden. ${ }^{38}$

30 Zeugenbefragung von Melech Gold am 26. Dezember 1983. YVA TR 11/PA 134, Bd. G.

31 Vgl. die Aussage von Fritz Hamann, der als Soldat der 96. I.D. die Morde in Paneriai erlebte: EK 3 Verfahren, Bd. 3, Bl. 1275, Vernehmung von Fritz Hamann vom 5. Juni 1959.

32 Vgl. Nementschik, Fort, S. 58ff. YVA TR 11/PA 134, Aussage von Jakob Gidansky vom 25. Mai 1982.

${ }^{33}$ Der erste Pogrom in Slobodka, dem mehrere Hundert Juden zum Opfer fielen, fand in der Nacht vom 25. auf 26. Juni 1941 statt. Vgl. Gringauz, Hurbn 7, S. 11.

${ }^{34}$ EK 3 Verfahren, Bd. 16, Bl. 5776, Vernehmung von Dr. Nikolai Löwenstein vom 12. Februar 1960.

${ }^{35} \mathrm{Vgl}$. zu den Vorgängen Dieckmann, Besatzungspolitik, 1, S. $333 \mathrm{ff}$.

${ }^{36}$ Vgl. LCVA R-643, ap. 3, b. 4152, Bl. 55 Rückseite, Vilniaus Miesto žydų komitetas [Jüdisches Komitee der Stadt Vilnius], Memorandum vom 24. Juli 1941 (Übersetzung aus dem Litauischen).

37 Vgl. LCVA R-689, ap. 3, b. 596, Bl. 3ff. Der Gefreite namens Schneider, der Sudorgas durch die litauische Polizei festnehmen ließ, war für die Bewachung der Juden zuständig. In seiner Zeugenaussage berichtete er, dass mehrere der geschlagenen Juden bluteten. Das Strafmaß nahm offensichtlich darauf Rücksicht, dass Sudorgas vollständig betrunken war. Eine weitere Motivsuche unternahmen die litauischen Polizisten nicht. Vgl. dazu auch Kruk, Last Days, S. 60, S. 68.

${ }^{38}$ Vgl. Shalit, Azoy, S. 20. Der Vorschlag wurde mit der Bemerkung abgelehnt, es handele sich dabei nicht um Selbstschutz, sondern um Selbstmord. Man einigte sich darauf, eine Delegation zur örtlichen sowjetischen Parteiführung zu entsenden, die darauf drängen sollte, dass die Juden aus Šiauliai evakuiert würden. 
Die völlige Schutzlosigkeit selbst in den eigenen vier Wänden prägte sich tief in die Erinnerung ein: „In der Nacht umstellten litauische Polizisten die Häuser, holten die Bewohner heraus, nahmen die Männer mit und jagten die Frauen und Kinder auf den Hof oder die Straße. Nur das, was man gerade in der Hand trug, durfte mitgenommen werden. Damit begann das Unheil für die jüdische Bevölkerung. Oft konnte man in der Nacht das Weinen und Schreien der Kinder in den Nachbarhäusern hören, in denen Haussuchungen gehalten wurden. "39 So war das litauische Verhalten auch ein Grund, dass die Juden der Ghettoisierung rasch zustimmten, denn ein abgesperrtes jüdisches Wohnareal verhieß immerhin Schutz vor dem litauischen Mob. ${ }^{40}$ Ein litauischer Gesprächspartner in Kaunas, der designierte Finanzminister der provisorischen Regierung Matulionis, erläuterte den Juden seine Sicht der Dinge, nach der es unter den Litauern drei Meinungen gebe, wie man mit den Juden verfahren solle: Die radikalste sei die der Ausrottung oder als Alternative die eines Konzentrationslagers mit einer entsprechend harten Behandlung. Er als gläubiger Katholik meine, man dürfe nicht töten, weswegen er für eine Trennung der Juden von den Litauern durch Ghettos sei. ${ }^{41}$ Für die Opfer hatte es oft den Anschein, als ob die Litauer aus eigenem Antrieb handelten. Der Chronist des Ghettos Šiauliai, Eliezer Jeruschalmi, sah z. B. die Erfassung der jüdischen Frauen und Kinder in Lagern im Gebiet um die Stadt als ausschließlich litauische Maßnahme an. ${ }^{42}$ Als Begründung für die brutalen Aktionen wurde von litauischer Seite immer auf die Taten der Bolschewisten, bei den Mördern auf Tote in der eigenen Familie verwiesen, um das Tun zu rechtfertigen. ${ }^{43}$ Die Deutschen nahmen solche Argumente gerne auf, so wurde den Juden in Kaunas erklärt, die Ghettoisierung habe auch deswegen zu erfolgen, weil die Litauer nicht mehr mit Juden zusammenleben wollten ${ }^{44}$, die sie allesamt

39 EK 3 Verfahren, Bd. 4, Bl. 1559, deutsche Übersetzung aus dem Buch „Geopfertes Volk - der Untergang des polnischen Judentums" von M. Chevasztein. Vgl. auch die Schilderung von Balberyszski, Shtarker, S. 233ff, bes. S. 235f, zur Liquidierung des Ghettos II in Vilnius.

${ }^{40}$ Vgl. Goldberg, Bletler, S. 35.

${ }^{41}$ Vgl. Tory, Surviving, S. 13.

42 Vgl. Jeruschalmi, Umkum, S. 1828. Jersuschalmi betont, die Litauer seien bis zum Auftauchen der deutschen Zivilverwaltung der Meinung gewesen, sie würden in Litauen bestimmen können.

${ }^{43}$ Vgl. die Aussage von Fritz Hamann, der als Soldat der 96. I.D. die Morde in Paneriai erlebte und mit dem litauischen Anführer ein Gespräch führte. Vgl. EK 3 Verfahren, Bd. 3, Bl. 1267, Vernehmung von Fritz Hamann am 5. Juni 1959. Auch wenn Hamann selbst dem Wahrheitsgehalt dieser Erklärung skeptisch gegenüberstand, bleibt festzuhalten, dass das Rachemotiv in Zusammenhang mit vielen Tätern Erwähnung findet. Erich Wolff, als Mitglied des EK 3 selbst zu den Tätern gehörend, machte bei seiner Vernehmung im April 1960 ebenfalls geltend, die Litauer hätten sich an den Juden wegen ihres Verhaltens während der Sowjetherrschaft gerächt und weil die Juden zu den Polen (gemeint ist offenbar der polnisch-litauische Streit um die Zugehörigkeit von Vilnius) gehalten hätten. Vgl. EK 3 Verfahren, Bd. 18, Bl. 6194, Vernehmung von Erich Willi Wolff vom 13. April 1960. Einer der deutschen Haupttäter in Vilnius, Horst Schweinberger, versuchte nach dem Krieg ebenfalls, alle Schuld auf die Litauer abzuwälzen: Vgl. EK 3 Verfahren, Band 23, Bl. 7288ff, Vernehmung von Polizeiobermeister Horst Schweinberger am 2. Dezember 1960.

${ }^{44}$ Die Bitte der Juden, mit den Litauern in Kontakt treten zu können, wurde von deutscher Seite mit der Begründung abgelehnt, kein Litauer wolle mit einem Juden sprechen. Vgl. Tory, Surviving, S. 14. Zu den Vorgängen in Kaunas vgl. S. 73f. dieser Arbeit. 
für Kommunisten hielten. ${ }^{45}$ Insofern konnte man im Ghetto die Illusion des Überlebens hegen, während außerhalb des Ghettos der sichere Tod wartete. ${ }^{46}$

Die ersten Tage der deutschen Besatzung waren neben dem Verhalten der Täter durch eine ganz bestimmte Art von Passivität großer Teile der litauischen Bevölkerung gekennzeichnet. Dazu gehörte auch der Erzbischof von Kaunas Juozopas Skvireckas, der der Bitte um Hilfe für Juden nicht nachkam. ${ }^{47}$ In Švenčionys sahen Einwohner teilnahmslos zu, wie jüdische Frauen und Kinder von litauischen Einheiten aus den Häusern getrieben wurden. ${ }^{48}$ Auch der litauische Stadtkommandant von Kaunas, Bobelis, der von dem ihm bekannten ehemaligen Offizier der litauischen Armee Goldberg während der Pogrome um Hilfe für die Juden gebeten wurde, sah sich außer Stande einzugreifen: Diese Angelegenheit, so der Oberst, liege weder in seinen Händen noch in seiner Kompetenz. ${ }^{49}$ Der langjährige frühere Bürgermeister von Kaunas Jonas Vileišis meinte gar, die litauische Jugend werde, wenn sie sich noch ein wenig ausgetobt habe, von selbst zur Ruhe kommen. ${ }^{50}$ So verdichtete sich der jüdische Eindruck immer mehr, dass die litauische Führungsschicht in keinster Weise daran interessiert war, den Pogromen und Ausschreitungen der ersten Tage Einhalt zu gebieten. ${ }^{51}$

Ein weiteres Beispiel, bei dem die Juden unter litauischer Willkür zu leiden hatten, betraf den Standort des Ghettos in Kaunas. Wie berichtet ${ }^{52}$, waren die Juden aus vielerlei Gründen mit Vilijampolè unzufrieden und wandten sich deshalb an die Sicherheitspolizei. Der Chef des EK 3 Jäger bekundete seinerseits sein Desinteresse am endgültigen Standort des Ghettos und verwies die jüdische Delegation an den litauischen Bürgermeister von Kaunas Kazys Palčiauskas. Dieser war jedoch zu keinerlei Zugeständnissen oder Entgegenkommen bereit, so dass das Ghetto in einem Stadtteil errichtet wurde, der nur über kleine Holzhütten und

${ }^{45}$ Vgl. Gar, Umkum, S. 44; Tory, Surviving, S. 13.

${ }^{46}$ Paraphrasierung nach der Formulierung von Jeruschalmi, Umkum, S. 1825. „In geto hat noch a jid gehobt die ilusie, az er ken bleibn leben, oisern geto is for in gewen a sicherer toit."

471959 berichtete dies der ehemalige Militärrabbiner der litauischen Armee S.A. Snieg den Ermittlern der Zentralen Stelle Ludwigsburg: Vgl. EK 3 Verfahren, Bd. 3, Bl. 1063, Vernehmung des Oberrabbiners S.A. Snieg am 3. Juni 1959. Hierzu auch der Bericht von Goldberg, Bletler, S. 32f, der vom Weihbischof Vincentas Brizgys empfangen wurde. Auf die Frage, wie die Kirche die Pogrome zulassen könne, und die Bitte, einen Bischofsbrief zu verkünden, habe der Bischof geantwortet, er könne nur wiederholen, dass die katholische Kirche nicht helfen könne. Vgl. Goldberg, Bletler, S. 32. Ein etwas positiveres Urteil über die Rolle der hohen litauischen Geistlichkeit fällt Šimaitè, Litwiner, S. 1664.

48 Vgl. Kaczerginski, Hurbn, S. 116.

${ }^{49}$ Vgl. Goldberg, Bletler, S.30f.; Tory, Surviving, S.11. Anicas, Bobelis, S. 182 ff., erwähnt in seiner Biographie des Stadtkommandanten die Begegnung nicht.

${ }^{50}$ Vgl. Goldberg, Bletler, S.31. Diese Begebenheit auch bei Dieckmann, Besatzungspolitik, 1, S. $331 \mathrm{f}$.

${ }^{51}$ So Goldberg, Bletler, S. 32, der meint, bei allen seinen Besuchen bei führenden Vertretern der litauischen Bevölkerung habe er immer wieder nur gehört, man könne nichts tun. Auch der jüdische Versuch, einen beständigen Kontakt mit litauischen Offiziellen herzustellen, von dem Tory, Surviving, S. 13, berichtet, führte zu keinem Erfolg. Jeruschalmi, Umkum, S. 1829, betont ausdrücklich die große Schuld der litauischen Elite an den Geschehnissen in und um Šiauliai.

52 Vgl. S. 75 dieser Arbeit. 
keine Kanalisation verfügte. ${ }^{53}$ Bitter kommentierte Avraham Tory in einer Ausarbeitung für die israelische Staatsanwaltschaft, die litauische Stadtverwaltung in Kaunas habe die konstruktive Zusammenarbeit der jüdischen Gemeinde zum Wohle der Stadt während der 1920er und 1930er Jahre völlig vergessen und Palčiauskas ${ }^{54}$ habe keine Spur von Solidarität und Kooperation gezeigt. ${ }^{55}$

In Vilnius ließ es sich die Stadtverwaltung angelegen sein, die Straßennamen aus der Zeit vor der sowjetischen Besatzung wieder einzuführen. Es gab jedoch Ausnahmen: „Von den bis zum 15. Juni 1940 gültigen Benennungen mußten wir z. B. diejenigen ausschalten, die ihre Namen von den Juden hergenommen haben/ also Gaon-Str./Schwarz-Str. u. a.m. ${ }^{\text {" }} 6$ Das städtische Standesamt legte besonderen Wert darauf, dass die Juden im Ghetto ein eigenes Amt einrichteten, denn „es ergebe sich aus dem Prinzip der gegenwärtigen Ordnung, dass Arier keine Juden bedienen" ${ }^{57}$ Und schließlich teilte die litauische Verwaltung dem jüdischen Komitee mit, die Lebensmittelkarten der in den ersten Tagen auf den StraBen festgenommenen Juden seien der städtischen Handelsabteilung abzuliefern, ein Missbrauch werde nach Kriegsrecht bestraft. ${ }^{58}$ Aus dieser Anweisung ergab sich zwangsläufig, dass mit einer raschen Rückkehr der Menschen nicht zu rechnen war, vielmehr das Schlimmste angenommen werden musste.

Ebenfalls aus den ersten Kriegstagen stammt folgender Bericht, der das litauische Verhalten beim Arbeitseinsatz einer willkürlich zusammengewürfelten Arbeitsgruppe in Vilnius schildert: „Für jeden Waggon wurden acht Mann eingeteilt, um den Zement auszuladen. Litauische Zivilisten mit Gummiknüppeln hetzten uns bei der Arbeit und schlugen uns rücksichtslos ins Gesicht und auf die Beine, wenn wir nicht schnell genug arbeiteten. Das Schleppen der schweren Zementsäcke erschöpfte bald alle bei dieser Hast und dem Durcheinander. Aus halber Betäubung jagte man uns durch Prügel wieder auf. Alle Gesichter waren von Überanstrengung und Entsetzen verzerrt. Als wir nach Hause zurückkehren durften, war die Polizeistunde schon vorbei. Um nicht erschossen zu werden, mar-

${ }^{53}$ Vgl. den Bericht von Goldberg, Bletler, S. 36. Hierbei soll Palčiauskas eine besonders üble Rolle gespielt haben, indem er den jüdischen Bitten nicht nur nicht entsprach, sondern das Ghettogelände noch verkleinerte. Vgl. den undatierten Investigator's Report mit Berufung auf Auskunft von Avraham Tory, Bl. 31, Bl. 64ff (YAV TR 11-1671 Kazys Palciauskas).

54 Palčiauskas war vom 25. Juni 1941 bis 1. Mai 1942 Bürgermeister von Kaunas.

${ }^{55}$ Vgl. YVA TR 11-1671, Kazys Palciauskas, A. Tory, Lithuanians preceded German occupation forces in persecution and torture of Jews in the first days of occupation in Lithuania (mss.), Bl. 109. Der Bürgermeister hatte den Juden auch den Zutritt zu den Diensträumen der Stadtverwaltung verboten. Vgl. Tory, Surviving, S. 25.

${ }^{56}$ LCVA R-614, ap. 1, b. 146, Bl. 11, Stadtverwaltung Wilna an den Herrn Gebietskommissar der Stadt Wilna, Betr. Beschilderung der Straßen vom September 1941 (die Angabe des exakten Datums fehlt).

${ }^{57}$ LCVA R-643, ap. 3, b. 4152, Bl. 176, J. Audejjus, Metrikų vedejjas Ponui Vilniaus Miesto burmistrui [J. Audejus, Leiter des Standesamtes an den Herrn Bürgermeister der Stadt Vilnius] vom 9. September 1941 (Übersetzung aus dem Litauischen).

58 Vgl. LCVA R-643, ap. 3, b. 4152, Bl. 301, Vilniaus Miesto ir Srities Piliečiu Komiteto Vidaus Reikalų Valdytojas, Referentas J. Čiuberkis, Vilniaus Žydų Komitetui [Der Chef für innere Angelegenheiten des Bürgerkomitees der Stadt und des Kreises Vilnius, Referent J. Čiuberkis an das Komitee der Juden von Vilnius] vom 7. August 1941. 
schierten wir in Viererreihen geschlossen nach Hause unter den Verwünschungen der Bevölkerung, die aus den Fenstern zusah. "59 Ähnliche Schilderungen zum litauischen Verhalten liegen aus Šiauliai vor, wo Juden auf offener Straße verprügelt wurden und nur wenige Litauer "einen Tropfen Menschlichkeit“ bewahrten. ${ }^{60}$ Verdächtigungen von Juden gehörten offenbar zum Alltag: In Vilnius brachten litauische Aufständische sechs Juden auf das 5. Polizeirevier, denen vorgeworfen wurde, sie hätten aus dem Fenster eines Hauses auf deutsche Soldaten geschossen. Obwohl die Polizisten nur eruieren konnten, dass in der Tat Schüsse auf die Soldaten abgegeben worden waren, aber keine Hinweise hatten, aus welchem Haus und Fenster das Feuer eröffnet worden war, wurden die Juden nach Lukiškès überstellt und der Kriegskommandant über den Vorfall informiert. ${ }^{61}$ Auch die Festnahmelisten des 4. Polizeireviers sprechen eine deutliche Sprache: Vom Tag des Einmarsches der Wehrmacht bis zum 4.Juli 1941 nahmen die Polizisten 41 Verhaftungen vor, von diesen Personen waren drei Litauer, drei Polen, sieben Russen und 28 Juden. ${ }^{62}$ Die meisten von ihnen wurden wegen kommunistischer Betätigung oder einfach mit der Begründung „Kommunist“ inhaftiert, was automatisch dazu führte, dass sie der litauischen Staatssicherheitspolizei überstellt wurden. ${ }^{63}$

So stehen die ersten Tage des deutsch-sowjetischen Krieges aus jüdischer Sicht auch für die schreckliche Erfahrung, dass die Juden auf keine Hilfe aus der benachbarten christlichen Welt hoffen konnten. ${ }^{64}$ In der litauischsprachigen Zeitschrift „I laisve““ [Zur Freiheit] des LAF erschien am 4. August 1941 ein Artikel mit dem Titel „Žydai sutvarkyti“ [Juden in Ordnung bringen, aufräumen - JT], der die Einrichtung von Ghettos und die strikte Trennung von Juden und Nichtjuden als neue Erfahrung für die Litauer beschrieb. Jetzt werde klar, wie sehr die Juden die Litauer ausgeplündert und unterdrückt hätten. Der Oberhexenmeister sei der Bolschewismus gewesen. ${ }^{65}$

59 EK 3 Verfahren, Bd. 4, Bl. 1557, deutsche Übersetzung aus dem Buch „Geopfertes Volk - der Untergang des polnischen Judentums“ von M. Chevasztein. Ob es sich bei der erwähnten Bevölkerung auch um Litauer handelte, muss offenbleiben.

60 Shalit, Azoy, S. 30: „ [...] a tropn menshlechkeit.“

${ }^{61}$ LCVA R-689, ap. 4, b. 916, Bl. 81, Vilniaus mst. policijos 5-tos nuovados Viršininkas Vilniaus Miesto Karo Vadui per Vilniaus mst. ir apskr. policijos Vadą [Der Vorsteher des 5. Reviers der Stadtpolizei Vilnius an den Kriegskommandanten der Stadt Vilnius über den Polizeichef von Stadt und Kreis Vilnius] vom 28. Juni 1941. Die Polizisten hatten zudem vergessen, die Personalien der Aufständischen aufzunehmen, so dass nur die Anwohner vernommen werden konnten, die wenig zur Aufklärung beitrugen.

62 LCVA R-689, ap. 4, b.916, Bl. 157 Vorder- und Rückseite, Vilniaus Miesto Policijos 4-tos nuovados veikimo žinios nuo pradžios iki $1941 \mathrm{~m}$. liepos mèn. $4 \mathrm{~d}$. 8 val. [Tätigkeitsbericht des 4. Polizeireviers der Stadt Vilnius vom Beginn bis zum 4. Juli 1941, 8.00 Uhr] vom 6. Juli 1941.

63 Vgl. den dementsprechenden Vermerk ebd., Bl. 157 Rückseite.

64 So Gar, Umkum, S. 289, der diese Erkenntnis in einer Liste von Argumenten für eine jüdische ,Selbstverwaltung;, d.h. das Leben in einem Ghetto, erwähnt, was noch einmal zeigt, wie tief die Gewalterfahrung der ersten Kriegstage das jüdisch-litauische Verhältnis (bis heute) belastet.

65 LCVA R-614, ap. 1, b. 145, Bl. 2. 
An entscheidenden Punkten der jüdischen Leidensgeschichte traten somit nicht die Deutschen, sondern die Litauer in Erscheinung. ${ }^{66}$ Hatten schon litauische Polizisten die Juden auf dem Weg zur Arbeit vor der Ghettoisierung beaufsichtigt, so waren sie auch in herausragender Weise am eigentlichen Transport der Juden ins Ghetto beteiligt. ${ }^{67}$ Bis zum zukünftigen Ghettotor wurden die Menschen nämlich von litauischen Polizisten bewacht. ${ }^{68}$ In Vilnius war die erste Nacht im Ghetto in besonderer Weise mit den litauischen Bewachern verbunden, denn die ganze Zeit hallten aufgeregte Schreie durch die Straßen, man solle das Feuer verlöschen. Gemeint war damit eine akribische Beachtung der Verdunkelungsbestimmungen, denn die Litauer vermuteten in jedem Lichtschein ein Signal der Juden an die Sowjets und drohten, das Feuer auf die Menschen zu eröffnen. ${ }^{69}$ Auch Jahre nach den Geschehnissen waren Überlebende der festen Überzeugung, die Litauer hätten damals eine weitgehende Autonomie beim Umgang mit Juden besessen. ${ }^{70}$

In Kaunas war es vor allem die litauische Stadtverwaltung, die, wie berichtet, alle Anregungen und Bitten des jüdischen Komitees im Zusammenhang mit der Ghettoisierung ablehnte. ${ }^{71}$ Als die Juden dann das Ghetto mit einem hohen Stacheldrahtzaun umgeben mussten, beaufsichtigten Beamte der Stadtverwaltung diese Arbeiten. ${ }^{72}$ Bei der ersten Aktion nach der Ghettoisierung, der sogenannten Intellektuellen-Aktion, die mehr als 500 Männern in Kaunas das Leben kostete, wurden die Menschen am Ghettotor von litauischen Partisanen in Empfang genommen, und auch die Lüge, man benötige für die Sichtung von Archiven gebildete Personen, wurde von litauischer Seite verbreitet. ${ }^{73}$ Da das Quorum aus Sicht der Gestapo noch nicht erfüllt war, kamen die Litauer ins Ghetto und trieben mit großer Brutalität weitere Opfer zusammen. ${ }^{74}$ Ähnliches spielte sich am Jom

${ }^{66}$ Eine Ausnahme findet sich in Gringauz, Hurbn 7, S. 8, wo es heißt, nur „einige lokale Anführer" hätten sich an den Judenmorden beteiligt.

${ }^{67}$ Vgl. die Aussage von Hercas Zusmanas in Vicas, SS Tarnyboje, S. 36.

${ }^{68}$ Vgl. LCVA R-1434, ap. 1, b. 1, Bl. 22, Der Gebietskommissar Wilna-Land Komisariniam Tarejui Ponui K. Kalendrai. Liečia: Žydų ịgyvendinima getuose [Der Gebietskommissar Wilna-Land an den Herrn komissarischen Rat K. Kalendra, betrifft: Die Ansiedlung der Juden in Ghettos] vom 19. September 1941. In diesem Schreiben ordnete Wulff an, in den Städten und Dörfern, wo es noch keine Ghettos gebe, diese schnellstens einzurichten. Dafür sowie für die Erfassung der Juden und ihre Begleitung ins Ghetto habe die litauische Hilfspolizei ihre gesamten Kräfte einzusetzen.

${ }^{69}$ Vgl. Balberyszski, Shtarker, S. 179; eine weitere Schilderung dieser Nacht bei Dworzecki, Yerusholajim, S. 68.

70 Vgl. LA SL Abt. 352 Lübeck, Nr. 1667, Protokoll der Aussage von Georg Fingerhut in den Räumen des Generalkonsulats der Bundesrepublik Deutschland in Montreal vom 28. März 1963, Bl. 168.

71 Vgl. Gar, Umkum, S. 281.

72 Vgl. Gar, Umkum, S. 48.

${ }^{73}$ Vgl. Gar, Umkum, S. 54f.; der litauische Beauftragte für jüdische Angelegenheiten hatte dem Ältestenrat berichtet, die leichte Arbeit in den Archiven sei für Intellektuelle besonders geeignet. Außerdem werde dadurch verhindert, dass die Menschen später zu schwerer körperlicher Arbeit herangezogen würden. Vgl. dazu Gringauz, Hurbn 7, S. 15.

${ }^{74}$ Vgl. Gar, Umkum, S. 55. 
Kippur im Ghetto in Vilnius ab. ${ }^{75}$ Und bereits bei der Ghettoisierung hatten sich die eingesetzten Litauer am 6. September 1941 wie „wilde Bestien“ verhalten, als sie die Juden aus den Häusern trieben. ${ }^{76}$

Hinzu kamen psychische Grausamkeiten und Erniedrigungen der Opfer. Bei der Aktion in Vilnius vom 24. Oktober 1941 wurden die ergriffenen Juden in der Rudnizker Straße gesammelt. Die sie bewachenden Litauer zwangen sie, das russische Liebeslied Katjuscha zu singen. ${ }^{77}$ Das Ghetto selbst sah aus wie eine einzige Ruine, überall hatten die Litauer aufgerissene oder eingetretene Türen und Schränke hinterlassen. ${ }^{78}$ Im Barackenlager bei Švenčionys suchten sich die litauischen Polizisten oft Mädchen aus, die die Nacht mit ihnen verbringen mussten. ${ }^{79}$ Am Tag der ,Großen Aktion', dem 28. Oktober 1941, bewachten in Kaunas neben deutschen Kräften auch litauische Polizisten die Juden. Außerdem fand sich eine größere Menschenmenge ein, um dem Spektakel zuzusehen. ${ }^{80}$

Eine besondere Rolle für das litauisch-jüdische Verhältnis spielten die litauischen Ghettowachen. ${ }^{81}$ „Schlimmer waren des öfteren die Litauer, die die Produkte abnahmen und in der Wache selbst aßen, oder wieder an Juden verkauften. "82 In Šiauliai galten die litauischen Wachen als "direkte Peiniger". ${ }^{83}$ Zwischenfälle zwischen den deutschen Begleitkommandos und der Torwache mussten diesen Eindruck zwangsläufig verstärken. ${ }^{84}$ Selbst die jüdische Ghettopolizei wurde von litauischen Wachen bedroht, wenn die jüdischen Polizisten versuchten, Handel über den Zaun hinweg zu unterbinden. ${ }^{85}$ Hinzu kamen Razzien

${ }^{75}$ Vgl. die Schilderung des Eindringens von zwei Litauern in die Wohnungen seines Wohnhauses bei Balberyszski, Shtarker, S. 217.

76 Balberyszski, Shtarker, S. 251. (Übersetzung aus dem Jiddischen)

77 Vgl. Balberyszski, Shtarker, S. 264f.

${ }^{78}$ Vgl. Balberyszski, Shtarker, S. 264. Bei der dreitägigen Aktion, die wenige Tage später erfolgte, nutzten die Litauer das Durchkämmen des Ghettos auch zu Räubereien. Vgl. Balberyszski, Shtarker, S. 286.

${ }^{79}$ Vgl. Kaczerginski, Hurbn, S. 117.

${ }^{80}$ Vgl. Vernehmung Zvi Levin vom 11. Juli 1982, YVA TR 11/PA 134.

${ }^{81}$ Auch die deutsche Seite sah Handlungsbedarf: Im Oktober 1941 untersagte die Führung des Reserve-Polizeibataillons 11 den litauischen Polizisten das Betreten des Ghettos ohne Vorliegen eines deutschen Befehls. Hintergrund waren die wiederholten Plünderungen sowie die Vergewaltigung einer Jüdin. Vgl. LCVA R-1444, ap.2, b. 5, Bl. 252 (litauischer Text) und Bl. 253 (deutscher Text), Res.Pol.Batl. 11 an den Verbindungsoffizier in Kauen, Betr.: Plünderungen im Ghetto durch Angehörige der lit. Btl. vom 18. Oktober 1941.

82 Vgl. EK 3 Verfahren, Bd. 3, Bl. 826f., Aussage Gustav Hörmann vom 2. September 1946 (Abschrift). Balberyszski, Shtarker, S. 199, berichtet, in den ersten Tagen des Ghettos sei der Schmuggel von Lebensmitteln noch relativ einfach gewesen, doch auch zu dieser Zeit hing dies "natürlich" bereits davon ab, welcher Litauer zur Torwache eingeteilt war. Selbst den Deutschen fiel das Verhalten der Litauer auf. So berichtete der Chef des EK 3 Jäger, die litauischen Wacheinheiten des Ghettos Kaunas hätten sich nicht bewährt: „ [...] es kam wiederholt zu Plünderungen und Überfällen auf Juden." BAB R 90/146, Einsatzkommando 3/A, Bl. 272.

${ }^{83}$ Shalit, Azoy, S. 91: „direkte Poiniker“.

${ }^{84}$ Vgl. LCVA R-643, ap. 3, b. 194, Bl. 143 Vorder- und Rückseite, Stadtverwaltung Wilna, Referent für Judenangelegenheiten an den Herrn Gebietskommissar der Stadt Wilna vom 8. Januar 1942.

${ }^{85}$ Vgl. LCVA R-973, ap. 2, b. 45, Bl. 5, Jüdische Ghettopolizei Vilijampolè an den Herrn Kommandanten der Deutschen Ghettowache vom 15. November 1941. 
im Ghetto, an denen immer litauische Einheiten beteiligt waren. ${ }^{86}$ Hierbei ging es den eingesetzten Polizisten häufig um ihre persönliche Bereicherung. ${ }^{87}$ Selbst im Ghetto vergriffen sich Einheimische an den Menschen, wobei die Täter sich offenbar durch die Beraubung der rechtlosen Juden eine besonders leichte Beute versprachen. Die Stadtverwaltung in Vilnius reagierte mit der Forderung, Wachen entlang des Ghettos patrouillieren zu lassen. ${ }^{88}$ In Kaunas vermerkte die Ghettopolizei in ihrem Bericht für Dezember 1942 lakonisch: „Im Berichtsmonat wurde das Ghetto wiederum von Verbrechern von jenseits des Zaunes heimgesucht, die Einbruchsdiebstähle in unweit des Zaunes gelegenen Höfen verübten. Die Art und das Ausmaß der Verbrechen läßt auf das Wirken einer organisierten Bande schließen."89 Zumindest ein Raubmord im Januar 1942 dürfte aufgrund der Zeugenaussagen von litauischen Unifomierten verübt worden sein. ${ }^{90}$ Auch Vergewaltigungen sind dokumentiert. ${ }^{91}$ Im April $1943 \mathrm{kam}$ es zu einer regelrechten Einbruchsserie, „deren Spuren“, so die vielsagende Bemerkung der Ghettopolizei, „meistens zum Ghettozaune führen“92 Die Täter sammelten sich offen am Ghettozaun, teilweise bestand die Menge aus Uniformierten und Zivilisten. ${ }^{93}$ Hilflos waren die Menschen auch, als ihnen litauische Kinder die Fensterscheiben

${ }^{86}$ EK 3 Verfahren, Bd. 4, Bl. 1575, deutsche Übersetzung aus dem Buch „Geopfertes Volk - der Untergang des polnischen Judentums“ von M. Chevasztein, zu den Ereignissen am Jom Kippur des Jahres 1941 im Ghetto Vilnius: „ [...] schon hörte man die Schreie der Frauen: ,Die Litauer sind im Ghetto! ““

87 Das Verhalten zweier junger Litauer, das Balberyszski, Shtarker, S. 236, am eigenen Leib erfahren musste, stellte sicher keine Ausnahme dar, zumal später immer mehr Litauer auf der Suche nach Geld auftauchten.

88 Vgl. LCVA R-643, ap. 3, b. 194, Bl. 167, Stadtverwaltung Wilna, Referent für Judenangelegenheiten an den Herrn Gebietskommissar der Stadt Wilna, Betr.: Wiederherstellung der Streifwache (Patrouille) um das jüdische Ghetto vom 13. Dezember 1941.

${ }^{89}$ LCVA R-973, ap. 2, b. 37, Bl. 2, Entwurf zum Bericht des Ältestenrates betr. Polizei, Dezember 1942. Vgl. auch LCVA R-973, ap. 2, b. 37, Bl. 527, Entwurf zum Bericht des Ältestenrates betr. Polizei, Oktober 1942. Einen Einblick in das Geschehen bieten auch die Wochenberichte der Ghettopolizei. In drei aufeinanderfolgenden Nächten drangen z. B. im Sommer 1942 zwei bewaffnete Litauer in das Ghetto ein und beraubten jüdische Bewohner. Vgl. LCVA R-973, ap. 2, b. 35, Bl. 577f., Seniūnų Tarybos Pirmininkui Savaites Žinios [Wochennachricht an den Vorsitzenden des Ältestenrates] für den Zeitraum vom 29. Juni bis 5. Juli 1942.

${ }^{90}$ LCVA R-973, ap. 2, b. 46, Bl. 118ff, Jüdische Ghettopolizei an den Herrn Kommandanten der Deutschen Torwache, Bericht Nr. 1 über den am 8. Januar 1942 in Vilijampolè, Jurgisstr. 11, erfolgten Raubmord.

${ }^{91}$ Vgl. LCVA R-973, ap. 2, b. 47, Bl. 12ff, Jüdische Ghettopolizei an den Herrn Chef der Ghettowache vom 15. Oktober 1941.

92 LCVA R-973, ap. 2, b. 41, Bl. 23 Rückseite, Bericht Nr. 19 über die Tätigkeit der jüdischen Ghettopolizei. April 1943. Dazu und zu Ausplünderungen von Juden weitere Fälle in LCVA R-973, ap. 2, b. 47, passim. Das Konvolut trägt den Vermerk „Byla apie ivykusius žydų apiplešimus" [Akte über erfolgte Ausplünderungen von Juden]. Die Fälle stammen aus dem Jahre 1941.

93 Vgl. LCVA R-973, ap. 2, b. 32, Bl. 94, Žydų Ghetto Policija, Centro İstaiga Seniūnų Tarybos Pirmininkui Raportas [Jüdische Ghettopolizei, Zentralamt, Bericht an den Vorsitzenden des Judenrates] vom 15. Dezember 1941. In dem Bericht wird ausdrücklich festgehalten, dass es sich bei den Personen um „Menschen litauischer Volkszugehörigkeit“" (Übersetzung aus dem Litauischen) gehandelt habe. Dazu auch der hss. Bericht der Ghettopolizei an die deutsche Ghettowache, in: LCVA R-973, ap. 2, b. 32, Bl. 102, Jüdische Ghettopolizei an den Herrn Kom- 
einwarfen: Man konnte nur die Ghettopolizei informieren und um Abhilfe bitten. ${ }^{94}$ in Vilnius nahmen litauische Uniformierte den Juden ihre Arbeitsausweise $\mathrm{ab}$ und gaben sie nur gegen eine Geldzahlung wieder zurück. ${ }^{95}$

Die offiziellen Kontakte der Ghettos mit den Besatzern liefen immer über die litauische Verwaltung. Die Beraubung der Juden erfolgte daher formal, sieht man einmal von den durch die Deutschen erpressten sogenannten Kontributionen der ersten Wochen $\mathrm{ab}$, durch die litauische Verwaltung und nicht durch die deutschen Besatzer. ${ }^{96}$ Aus den Berichten von Buragas und Gens geht deutlich hervor, dass die deutsche Seite vor allem am Arbeitseinsatz interessiert war. ${ }^{97}$ Ebenso musste sich in Kaunas das jüdische Komitee nach der Abschließung des Ghettos in Ernährungsfragen an die litauische Seite wenden. ${ }^{98}$ Und wie in Vilnius war auch hier ein Litauer, nämlich ein früherer höherer Beamter des litauischen Finanzministeriums namens Kaminskas, der Ansprechpartner des Judenrates. ${ }^{99}$ In Šiauliai verlief der Instanzenweg ebenfalls über die litauische Stadtverwaltung, der dortige Judenreferent Stankus ${ }^{100}$ war Hauptmann in der litauischen Armee gewesen. ${ }^{101}$

Beharrlich versuchte die litauische Bürokratie, ,Judenschulden', die vor dem 22. Juni 1941 aufgelaufen waren, einzutreiben ${ }^{102}$, zumal die Juden als lukrative Zahler galten: „Schon in meinen früheren Berichten habe ich angegeben, dass vor

mandanten der Deutschen Ghettowache Vilijampolè, undatiert. Ein weiteres Beispiel vom 12. Oktober 1941 ebd., Bl. 382.

94 Vgl. LCVA R-973, ap. 2, b. 46, Bl. 44, Mitteilungs-Protokoll vom 28. Juli 1942.

95 Vgl. Kruk, Last Days, S. 468.

96 Vgl. LCVA R-643, ap. 3, b. 4152, Bl. 127, Der Gebietskommissar der Stadt Wilna an die Stadtverwaltung in Wilna, z. Hd. des Referenten Buragas, Betr.: Anmeldung des jüdischen Vermögens vom 22. August 1941.

97 Vgl. z. B. LCVA R-643, ap. 3, b. 194, Bl. 3, Referent für Judenangelegenheiten an den Herrn Gebietskommissar in Wilna vom 29. September 1942, Betr.: Ghetto-Monatsbericht für September. Dieser Bericht umfasst mehrere Anlagen zu den Arbeitsstellen und der Auftragslage der Ghettowerkstätten, darüber hinaus wird in wenigen Worten nur über die Zahl der Ghettobewohner und die Größe des Ghettos berichtet. Die litauische Vorlage des Berichts ebd., Bl. 7, Vilniaus geto pirmūnas ir policijos vadas Ponui Žydų reikalams referentui [Der Ghettovorsteher und Polizeichef an den Herrn Judenreferenten] vom 26. September 1942.

98 Vgl. Goldberg, Bletler, S. 41. Man wandte sich an Jonas Matulionis, den Wirtschaftsminister der provisorischen Regierung, der den Juden eine menschliche Ernährung zusagte. Allerdings war der Einfluss der provisorischen Regierung in der kurzen Zeit ihrer Existenz sehr begrenzt.

99 Vgl. Goldberg, Bletler, S. 42; Tory, Surviving, S. 25f. Kaminskas' erster Auftritt vor dem Ältestenrat erfolgte, als er dem Gremium den Befehl des Stadtkommissars Cramer überbrachte, sich am 9. August vollzählig bei ihm zu melden. Bald danach gab er die Aufforderung, 500 gebildete Personen für Archiv- und Bibliothekssichtungen zu stellen, weiter - und die erste Massenmord-Aktion in Kaunas begann.

100 Vgl. Samuel Burgin an die Zentrale Stelle der Landesjustizverwaltungen vom 21. Januar 1960. LA SL Abt. 352 Lübeck, Nr. 1664, Bl. 50; Samuel Burgin, Eidesstattliche Erklärung am 25. Juli 1960. LA SL Abt. 352 Lübeck, Nr. 1664, Bl.145. Vgl. dazu auch die Zeugenaussage von Aron Abramson, ZS Ludwigsburg AR-Z 774/1961, Gewecke Hans, Bl. 360, vom 9. Februar 1965. Zu Stankus daneben die Anekdote bei Jeruschalmi, Umkum, S. 1778.

101 Vgl. die Aussage von Aron Abramson vom 2. Oktober 1969 in LA SL Abt. 352 Lübeck, Nr. 1690, Bl. 15f, Bl. 19f.

102 Dazu ausführlich Tauber, Verwaltung, S. $103 \mathrm{ff}$. 
dem 22. Juni 1941 Juden als Besitzer der Betriebe, Verkaufsgeschäfte, Häuser usw. wichtigste Steuerzahler der Stadtverwaltung waren. Die Juden sind der Stadtverwaltung insgesamt ca. 520000 RM schuldig geblieben, ohne Verzugsstrafen hinzuzurechnen. Mit Rücksicht auf schwere Lage der Stadtkasse wäre es wünschenswert, diese Judenschulden baldigst zu bekommen. " 103 Auch die sogenannte Miete, die die Juden für die ,Benutzung' des Ghettos entrichten mussten, ging in Vilnius an die Stadtverwaltung. ${ }^{104}$

In manchen Fällen gebärdeten sich die Litauer deutscher als die Deutschen. Der Referent für Judenangelegenheiten der Stadtverwaltung Vilnius machte seinen deutschen Vorgesetzten Murer auf folgenden Sachverhalt aufmerksam: „Bei Kontrollierung einzelner in der Stadt herumgehenden Juden hat sich herausgestellt, dass die leitenden Stellen der Arbeitsstätten und die Geleitwachen der Judenkolonnen den Verkehr der Juden mit der Bevölkerung bewußt begünstigen, indem sie einzelne Juden in die Stadt zum Einkauf der Lebensmittel herauslassen oder die Trennung der Juden von den nach der Arbeit ins Ghetto zurückmarschierenden Kolonnen gestatten. So gelingt es den Juden, ununterbrochene Verbindung mit den Preiswucherern aufrecht zu erhalten, und es ist nicht zu verwundern, dass sowohl die Kolonnen als auch einzelne Juden öfters mit Lebensmitteln beladen ins Ghetto zurückkehren. " 105 Auch nach der Stabilisierung der Lage kam es öfter zu unliebsamen Erfahrungen mit Litauern. Viele Juden, die in den Stadtbrigaden arbeiteten, wurden von litauischen Polizisten bei ihren Versorgungsgängen entdeckt und oft direkt der Gestapo übergeben. ${ }^{106}$ Die vier städtischen Bibliotheken wurden offensichtlich ohne deutsche Anweisungen einer sprachlich-ideologisch-rassistischen Säuberung unterzogen. ${ }^{107}$

Oskar Schönbrunner, einer der wenigen deutschen Judenretter, machte in Vilnius folgende Beobachtung: „Die litauische und polnische Bevölkerung widersprach den Erschießungen nicht. Ich hatte den Eindruck, sie sei antisemitisch eingestellt. Ob diese Einstellung von Anfang an vorhanden war oder erst durch nationalsozialistische Einflüsse hervorgerufen wurde, entzieht sich meiner Kennt-

103 LCVA R-614, ap. 1, b. 732, Bl. 31, Miesto savivaldybè, Sekretoriatas [Stadtverwaltung, Sekretariat] an den Herrn Gebietskommissar der Stadt Wilna, betr.: Finanzielle Lage der Stadtverwaltung Wilna vom 31. Dezember 1941.

104 Vgl. S. 208, Anm. 696 dieser Arbeit.

105 LCVA R-643, ap. 3, b. 194, Bl. 143ff, Stadtverwaltung Wilna, Referent für Judenangelegenheiten an den Herrn Gebietskommissar der Stadt Wilna vom 8. Januar 1942. Buragas schlug vor, den Arbeitgebern zu untersagen, die Juden mit irgendwelchen Bescheinigungen in der Stadt herumzuschicken bzw. die entsprechenden Ausweise über das Arbeitsamt auszustellen. Außerdem sollte ein Angehöriger der SS an der Torwache anwesend sein, um Unstimmigkeiten zwischen den Begleitkommandos der Brigaden und der (litauischen) Torwache zu vermeiden. Zumindest der erste Vorschlag wurde in Vilnius umgesetzt.

106 Vgl. Gar, Umkum, S. 117.

107 Vgl. LCVA R-643, ap. 3, b. 2903, Bl. 19 Rückseite, Leiter der Direktion für Bildungswesen an das Gebietskommissariat Wilna-Stadt, Referat Kultur und Erziehung vom 1. März 1943. Für die "Durchprüfung der privaten Bibliotheken“ hatte die Direktion spezielle Gruppen gebildet. Vgl. ebd., Bl. 20. Hintergrund der Aktion bei den privaten Bibliotheken war eine deutsche Anweisung. Zum Ergebnis der Überprüfung vgl. ebd., Bl. 49 vom 8. Juli 1943. 
nis. Jedenfalls schaute die Bevölkerung teilnahmslos zu, wenn Juden zur Hinrichtung getrieben wurden. "108 Die deutschen Besatzer in Kaunas verfügten in ihren Anordnungen sogar, die Juden seien vor Belästigungen und Willkür zu schützen, ein klarer Hinweis auf ein dementsprechendes Verhalten von Einheimischen. ${ }^{109}$

Ein weiteres Charakteristikum, das sich in jüdischen Berichten immer wieder findet, ist das der litauischen Treulosigkeit. ${ }^{110}$ Oft nutzten die Einheimischen die jüdische Notlage schamlos aus. Selbst Eltern, die ihre Kinder in Sicherheit bringen wollten, wurden Opfer solcher Machenschaften. Manchmal wurden die Kinder, für deren Aufnahme in ,arischen' Familien die jüdischen Eltern gezahlt hatten, nach einigen Tagen unter dem Vorwand, die Gestapo sei misstrauisch geworden, wieder ins Ghetto zurückgeschickt (das Geld wurde natürlich einbehalten). ${ }^{111}$ Und schließlich war der Handel mit den schutz- und hilflosen Juden für manche Litauer ein sehr lukratives Geschäft. In Šiauliai beteiligte sich die weibliche Landbevölkerung häufig an derlei Handel, der geradezu einer Naturalienwirtschaft glich: Für ein neues Bettlaken bekam man z. B. 1 kg Butter. ${ }^{112}$

Natürlich gab es auch andere Verhaltensweisen: In Mažeikiai weinte die Mutter des 16-jährigen Vytautas Bernotas, als sie erfuhr, dass ihre jüdischen Nachbarn ermordet worden waren. ${ }^{113}$ Abraham Sutzkever berichtet aus Vilnius, dass die deutsche antijüdische Hasspropaganda in breiten Teilen der einheimischen Bevölkerung ohne Resonanz geblieben sei. ${ }^{114}$ Die Beerdigung des Optikers Miron Abelowitz fand unter Teilnahme seines litauischen Chefs und weiteren Christen aus der Belegschaft im Ghetto statt. ${ }^{115}$ Ein litauischer Polizeibeamter warnte in Šiauliai die Juden vor Aktionen. ${ }^{116}$ Absolute Ausnahmen scheinen Kontakte zwischen Juden und Litauern im Zeichen eines Widerstandes gegen die Besatzer ge-

108 EK 3 Verfahren, Bd. 23, Bl. 7243, Vernehmung von Oskar Schönbrunner vom 21. November 1960.

109 Vgl. LCVA R-616, ap. 1, b. 11, Bl. 25, Der Stadtkommissar in Kauen, Ref. II: An alle Dienststellen, die Juden beschäftigen, vom 30. Juli 1942.

110 Balberyszski, Shtarker, S. 244f., schildert z. B. den Fall eines Litauers, der eine Maline im Ghetto II, von deren Lage er Kenntnis bekommen hatte, an die Gestapo verriet. Das Misstrauen gegen die Litauer hatte solche Ausmaße erreicht, dass einige Menschen die Maline nur auf den Verdacht, der Litauer könne sie verraten, verlassen hatten.

111 So Gar, Umkum, S. 170, der zudem berichtet, in einigen Fällen seien Kinder drei bis vier Mal auf diese Weise zwischen Ghetto und Stadt hin- und hergeschickt worden, bevor eine ehrliche Familie sich ihrer angenommen habe. Die Zahl derjenigen Litauer, die aus humanitären Gründen ein jüdisches Kind bei sich aufnahmen, sei dagegen nicht sehr groß gewesen. Dazu auch Eilati, Crossing, S. $104 \mathrm{ff}$.

112 So Shalit, Azoy, S. $67 \mathrm{f}$.

113 Vgl. EK 3 Verfahren, Bd. 5, Bl. 1875, Aussage von Vytautas Bernotas.

114 Vgl. Sutzkever, Geto, S. 80. Ob Sutzkever damit die polnisch- oder litauischsprachige Bevölkerung meint, muss offenbleiben, es spricht jedoch alles dafür, dass sich diese Aussage vor allem auf Letztere bezog.

115 Vgl. Kruk, Last Days, S. 492.

116 Samuel Burgin an die Zentrale Stelle der Landesjustizverwaltungen vom 21. Januar 1960. LA SL Abt. 352 Lübeck, Nr. 1664, Bl. 50, gibt den Namen des Polizisten mit Greblunas an. Zu weiteren Judenrettern vgl. Jeruschalmi, Umkum, S. 1830f. Šimaitè, Litwiner, S. 1663, berichtet von drei litauischen Polizisten, die in Vilnius den Juden halfen. 
wesen zu sein. ${ }^{117}$ Manchmal ergaben sich auch positive Beziehungen mit Einheimischen am Arbeitsplatz. ${ }^{118}$ Doch die große Mehrheit verhielt sich gegenüber dem jüdischen Schicksal passiv. ${ }^{119}$

Für diejenigen, die untertauchen wollten, waren gute litauische Sprachkenntnisse unabdingbar ${ }^{120}$, „a matter of life and death" ${ }^{121}$. Abgesehen davon spricht es für sich, dass die Überlebenden der Liquidierung des Ghettos in Vilnius offensichtlich keine andere Chance sahen, als sich in die noch bestehenden jüdischen Arbeitsstellen beim HKP und bei Kailis zu retten. ${ }^{122} \mathrm{Zu}$ feindlich erschien ihnen das ,arische“ Umfeld. „Ach, wissen Sie, die Polen und die Litauer konnten die Juden im Untergrund erkennen, auch wenn sie wie ich aschblond und blauäugig waren. Nicht durch die Farbe der Haare, Augen, die Form der Nase, sondern durch den Mangel an Dreistigkeit, an Selbstsicherheit, nicht durch den Akzent der Sprache, sondern durch die Art, sich auszudrücken, wurden sie erkannt."123 ,Litauisch' aussehende Frauen wurden daher vom jüdischen Widerstand bevorzugt als Kurierinnen eingesetzt. ${ }^{124}$

Über allen diesen Gegensätzen stand der fundamentale Dissens zwischen Litauern und Juden, was die jeweiligen Zukunftsvorstellungen betraf. Deutlich wird dies in Zusammenhang mit der sowjetischen Partisanenbewegung: Während für viele, vor allem junge Juden die Flucht in die Wälder als einziger Ausweg aus der Ghettosituation erschien, standen große Teile der litauischen Bevölkerung der Partisanenbewegung feindlich gegenüber. ${ }^{125}$ Das EK 3 berichtete zum jüdisch-litauischen Verhältnis: „Die litauische Bevölkerung brachte den Maßnahmen der deutschen Sicherheitspolizei wohlwollendes Verständnis entgegen." Allerdings

117 Selbst der SD wusste nur über Einzelfälle zu berichten. Vgl. LCVA, R-1399, ap. 1, b. 26, Bl. 108, Der Kommandeur der Sicherheitspolizei u. d. SD - Litauen - Geheim, Lagebericht 5/43 der Abteilungen III, IV und V: Lagebericht für die Zeit vom 1. bis 31.5.1943 vom 31. Mai 1943. Ginaite, Atminimo, S. 113, fand Unterstützung und Hilfe bei einer litauischen Familie, die sie seit Kindesbeinen kannte. Schließlich firmierte das Lädchen der Litauer als Kontaktstelle des Widerstandes.

118 Vgl. Kruk, Last Days, S. 79. Allerdings handelte es sich zumindest bei einem der beiden Arbeiter, die nett zu ihm waren, um einen Polen, während der andere nur als Christ bezeichnet wird.

119 Šimaite, Litwiner, S. 1665, mit der lakonischen Bemerkung, die meisten Litauer hätten den Juden weder Gutes noch Böses getan.

120 Balberyszski, Shtarker, S. 226, berichtet von einem Gespräch mit einem Bekannten, dessen junger Sohn in die Stadt geflohen war, weil er gut Litauisch sprach und sich im Untergrund eine bessere Überlebenschance erhoffte als im Ghetto.

121 Eilati, Crossing, S. 105.

122 Vgl. dazu mit weiteren Einzelheiten S. 365 dieser Arbeit.

123 Kruk, Schreie, S. 202. Faitelson, Widerstand, S. 174: „Die Deutschen konnten vielleicht einen Juden nicht von einem Nichtjuden unterscheiden, doch die Litauer ließen sich diesbezüglich nicht überlisten. Jedes litauische Kind konnte mit dem Finger auf einen von uns zeigen und schreien: ,Žydas' - ,Jude،“"

124 Vgl. dazu die Schilderungen von Ginaite, Atminimo, S. $111 \mathrm{f}$, die sich auch in ihrer Kleidung als „Mädchen aus Kaunas“ tarnte.

125 So die zutreffende Einschätzung bei Gar, Umkum, S. 144. 
musste man auch konzedieren, die „Unterstützung der einheimischen Bevölkerung“ habe „nachgelassen“. 126

Wie negativ die Litauer aus jüdischer Sicht erschienen, wird überdeutlich bei den Vorfällen um das kleine Ghetto in Swir, einem Städtchen, das im April 1942 vom GK Weißruthenien dem GK Litauen zugeschlagen worden war. Diejenigen Juden, die aus Litauen zuvor ins Swirer Ghetto geflohen waren, machten sich auf die Nachricht von der Übernahme durch die Litauer hin sofort wieder davon. ${ }^{127}$

Das litauische Verhalten unterlag offensichtlich teilweise auch der jeweiligen Konjunktur. Herman Kruk fiel im Winter 1941/42 auf, dass nach den deutschen Niederlagen vor Moskau und den sowjetischen Gegenoffensiven die litauischen Ghettowachen sich weniger energisch und pflichtbewusst gebärdeten. ${ }^{128}$ Und ein Jahr später notierte er, dass 23 Juden, die im berüchtigten Gefängnis Lukiškès inhaftiert waren, von einem geradezu freundlichen Verhalten des litauischen Gefängnispersonals zu berichten wussten. ${ }^{129}$

Auch nach dem Ende des Krieges entspannte sich das jüdisch-litauische Verhältnis nicht. Aus vielen Zeugnissen von Überlebenden spricht eine tiefe Verbitterung über das litauische Verhalten: „Hier brauchte man keinen Adolf Eichmann und Kolonnen von Güterzügen, um die Menschen in die Todesfabriken zu bringen. Es genügte ,ein Rollkommando von 10 bewährten Männern' (Jäger-Bericht) - das übrige besorgten Hunderte unserer langjährigen litauischen Nachbarn -, um die gesamte jüdische Bevölkerung der litauischen Provinz [...] im Verlauf von nur sechs Monaten auf grausamste Weise umzubringen. Die willigen Henker verrichteten ihre Arbeit so besessen und eifrig, dass Litauen zu einem der bevorzugten Plätze des Mordes [...] wurde."130

So nahmen die litauisch-jüdischen Beziehungen „tragisch-scharfe Formen“ an, die man sich, so Ona Šimaitè, eine litauische Judenretterin, vor dem Krieg überhaupt nicht hätte vorstellen können. ${ }^{131}$ Zugleich warnte sie bereits in ihrem 1952 erstmals erschienenen Bericht vor einer litauischen Kollektivschuld. ${ }^{132}$ In der Tat dürfte aufgrund der Ausführungen in diesem Kapitel deutlich geworden sein, dass die Prominenz von litauischen Tätern und Nutznießern der jüdischen Tragödie weder auf wenige Einzelpersonen eingegrenzt noch pauschal auf größere Bevölkerungsgruppen ausgedehnt werden kann. ${ }^{133}$

126 BAB R 90/146, Einsatzkommando 3/A, Verhältnis der litauischen Bevölkerung gegenüber den Juden. Als Erklärung für das schwindende Verständnis machte Jäger die litauische Intelligentsia aus, die versuche, die Bevölkerung zu einer mehr judenfreundlichen Haltung zu bewegen.

127 Vgl. Kaczerginski, Hurbn, S. 130. Das im Text erwähnte Datum der Umgliederung (April 1943) ist falsch.

128 Vgl. Kruk, Last Days, S. 151.

129 Vgl. Kruk, Last Days, S. 435.

130 Katz, Memel, S. 153. Ähnlich Margolis, Partisanin, S. 97.

131 Vgl. Šimaite, Litwiner, S. $1661 \mathrm{f}$.

132 Vgl. Šimaitè, Litwiner, S. 1670.

133 Hierzu Tauber, Vergangenheitsbewältigung, passim. 
Dennoch gehört die feindliche und ablehnende Haltung der litauischen Bevölkerung zu den zentralen Erinnerungsmotiven der Ghettophase und des damit verbundenen Arbeitseinsatzes. Nicht zuletzt daraus läßt sich die Affinität der jüdischen Arbeiter zu Arbeitsplätzen bei deutschen, bevorzugt militärischen Dienststellen erklären. 